Accepted in Astrophysical Journal(ApJ)

Preprint typeset using $\mathrm{IAT}_{\mathrm{E}} \mathrm{X}$ style emulateapj v. 12/16/11

\title{
ROLE OF ENVIRONMENT ON NUCLEAR ACTIVITY
}

\author{
Amirnezam Amiri \& SAeEd Tavasoli \\ Physics Dept., Kharazmi University, Tehran, Iran \\ Gianfranco De Zotti \\ INAF-Osservatorio Astronomico di Padova,Vicolo dell'Osservatorio 5, I-35122 Padova, Italy \\ Accepted in Astrophysical Journal(ApJ)
}

\begin{abstract}
Motivated by the apparently conflicting results reported in the literature on the effect of environment on nuclear activity, we have carried out a new analysis by comparing the fraction of galaxies hosting active galactic nuclei (AGNs) in the most overdense regions (rich galaxy clusters) and the most underdense ones (voids) in the local universe. Exploiting the classical BPT diagnostics, we have extracted volume limited samples of star forming and AGN galaxies. We find that, at variance with star-forming galaxies, AGN galaxies have similar distributions of specific star formation rates and of galactic ages (as indicated by the $\mathrm{D}_{n} 4000$ parameter) both in clusters and in voids. In both environments galaxies hosting AGNs are generally old, with low star formation activity. The AGN fraction increases faster with stellar mass in clusters than in voids, especially above $10^{10.2} M_{\odot}$. Our results indicate that, in the local universe, the nuclear activity correlates with stellar mass and galaxy morphology and is weakly, if at all, affected by the local galaxy density.
\end{abstract}

Keywords: Void galaxy, Cluster galaxy, AGN activity, Stellar parameters

\section{INTRODUCTION}

It has long been known that, in the nearby universe, galaxy properties such as star formation rate (SFR), morphology and stellar mass are strongly correlated with the surrounding galaxy density (Oemler 1974: Dressler 1980 Hashimoto et al. 1998; Kauffmann et al. 2004: Baldry et al. 2006). Massive early-type galaxies in passive evolution are preferentially found in high density environments while the specific SFR increases towards lower density regions. This suggests that the environment has a substantial role in driving galaxy evolution, although the details of the physical processes involved are still poorly understood.

We focus here on the role of environment on nuclear activity. This is relevant to constrain processes that trigger and control it and to investigate its relationship to star formation. Several analyses have been carried out considering various definitions of the local density. It is generally agreed that the fraction of galaxies hosting active galactic nuclei (AGN) depends on stellar mass Pimbblet et al 2013 and references therein, but the dependence on environment at fixed stellar mass is debated.

Carter et al. (2001) claimed that the AGN fraction, $F_{\mathrm{AGN}}$, is insensitive to the environment. A similar conclusion was reached by Miller et al. (2003) who observed that while the fraction of star-forming galaxies decreases with density, the fraction of galaxies with an AGN remains constant from the dense cores of galaxy clusters to the low density field. Sabater et al. (2015) argued that the effect of the local density is minimal at fixed stellar mass and central star formation activity. This is in agreement with the analysis of Yang et al. (2018) who found that the sample-averaged accretion rate onto

amirnezamamiri@gmail.com super-massive black holes does not show any significant dependence on overdensity or cosmic-web environment once the stellar mass of host galaxies is controlled, and with the finding by Karhunen et al. (2014) that quasar environments are not significantly different from those of normal galaxies with similar luminosities.

In contrast, Kauffmann et al. (2004) reported a strong anti-correlation of nuclear activity on local density at fixed stellar mass for powerful AGNs ( $L([\mathrm{OIII}])$ > $10^{7} L_{\odot}$ ), generally residing in massive galaxies with significant star formation activity. The strong dependence of the prevalence of nuclear activity with density and interactions was found after taking into account the effect of mass. According to Constantin et al. (2008), however, such a trend holds only for moderately bright $\left(M_{\mathrm{r}} \approx\right.$ $-20)$, and moderately massive $\left(10<\log \left(M_{\star} / M_{\odot}\right)<\right.$ 10.5) galaxies; their data do not show any statistically significant excess of any type of AGN in the most massive void galaxies relative to those in relatively crowded regions ("walls"). Deng et al. (2012) found a different environmental dependence for low and high stellar mass galaxies. In the former $F_{\mathrm{AGN}}$ depends very little on local density but, in the latter, it decreases with increasing density. Lopes et al. (2017) confirmed these conclusions.

The opposite conclusion was reached by Manzer \& De Robertis (2014) who reported a significant increase in the AGN fraction in group environments compared with isolated galaxies. A larger fraction of AGN in denser environment was also found by Argudo-Fernández et al. (2018) for quenched and red galaxies.

A related issue is the role of interactions/mergers in triggering nuclear activity. This problem has been addressed in different ways. Schawinski et al. (2010) looked for major morphological disturbances in a sample of spheroidal galaxies in the process of migrating from the 


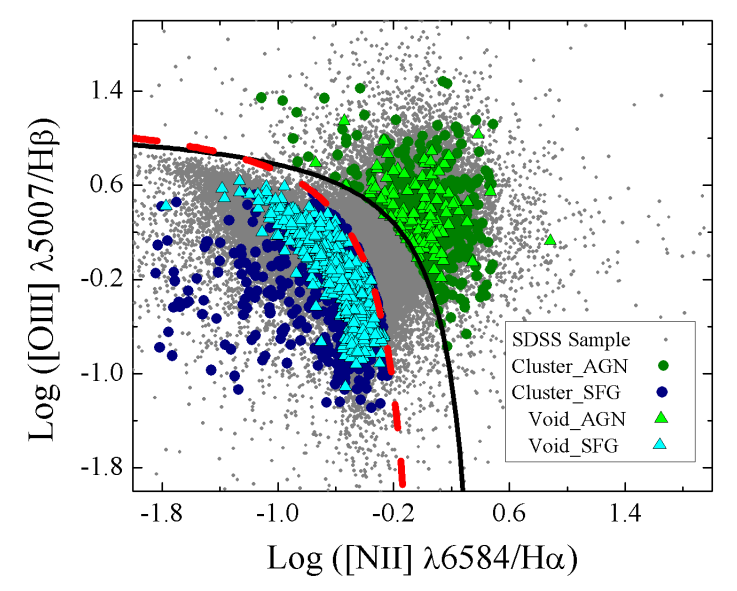

Figure 1. Diagnostic diagram to discriminate between starforming galaxies and galaxies hosting AGNs. The solid black line and the dashed red line show the separations between the two populations according to the Kewley et al. (2001) and the Kauffmann et al. (2003) criteria, respectively. Circles and triangles reter to cluster and vold galaxies, respectively. We have not classified galaxies located between the two lines.

blue cloud to the red sequence via an AGN phase. Other studies compared the AGN fraction in samples of close galaxy pairs with that in a control sample of galaxies with similar masses and redshifts but no nearby companion (Ellison et al. 2011, 2015, Satyapal et al. 2014; Scott et al. 2014). The general conclusion of these investigations is that interactions/mergers substantially enhance the specific SFR, but the effect on AGN activity depends on the AGN type. The AGN fraction was found to be substantially higher in close galaxy pairs (Ellison et al. 2011, 2015; Satyapal et al. 2014), implying that mergers can trigger nuclear activity and enhance the accretion rate. However, the fraction of low excitation radio galaxies (LERGs) was found to be independent of projected separation, implying that LERGs are not fuelled by mergers (Ellison et al. 2015). Scott et al. (2014) reported a modest decrease of the Seyfert fraction in close pair galaxies compared to isolated galaxies, suggesting that either mergers may not trigger AGN activity at the close pair stage or may trigger a different AGN type. Alternatively, the lack of a clear link between merger features and AGN activity may be due to the large time delay between the merger-driven starburst and the peak of AGN activity, allowing the merger features to decay (Schawinski et al. 2010).

The apparently contradictory results on the effect of environment on nuclear activity have motivated our reanalysis. In this paper, we compare the extremes of the density distribution of large scale structures: the most overdense regions (galaxy clusters) and the most underdense ones (voids). In Sect. 2, we present the samples used and describe their properties. The results of our analysis are reported in Sect. 3 while in Sect. 4 we summarize our main conclusions.

\section{SAMPLE SELECTION AND GALAXY CLASSIFICATION}

We have selected a volume-limited sample of void galaxies brighter than $\mathrm{M}_{r}=-18$ drawn from the void catalogue by Tavasoli et al. (2015). The sample covers the redshift range $0.01<z<0.04$, the maximum redshift being determined by the limiting absolute magnitude, and comprises 1159 galaxies.

Cluster galaxies in the same redshift and absolute magnitude range of void galaxies were extracted from the catalogue by Tempel et al. (2014), considering only clusters with at least 15 spectroscopic members. Brightest cluster galaxies (BCGs) themselves were excluded in consideration of their different evolution compared to the other member galaxies (Lauer et al. 2014). The final sample contains 2870 cluster galaxies.

To separate star-forming galaxies (SFGs) from galaxies hosting AGNs (hereafter AGNs) we exploited the classical BPT diagnostics (Baldwin et al. 1981) based on emission line ratios. The emission line intensities for both void and cluster galaxies were taken from the MaxPlanck-Institute for Astrophysics (MPA)-Johns Hopkins University (JHU) SDSS DR7 catalog (MPA/JHU; Kauffmann et al. 2003, Brinchmann et al. 2004) with $\mathrm{S} / \mathrm{N}>3$ for all objects. All the 1159 void galaxies and 2865 out of the 2870 cluster galaxies have emission lines and are included in the MPA/JHU catalog.

Figure 1 shows the distribution of our galaxies in the $[\mathrm{OIII}] 5007 / \mathrm{H} \beta$ versus the $[\mathrm{NII}] 6584 / \mathrm{H} \alpha$ diagram together with the boundary lines between SFGs and AGNs defined by Kewley et al. (2001) and by Kauffmann et al. (2003). The demarcation line by Kewley et al. (2001) is a theoretical upper limit on the location of SFGs in this diagram, obtained using a combination of photoionization and stellar population synthesis models. It yields a conservative AGN selection. Kauffmann et al. (2003) have revised the boundary line on the basis of the observational evidence that SFGs and AGNs are distributed along two separated sequences. It yields a conservative SFG selection.

In order to avoid ambiguous classifications we have adopted the more conservative selection for both SFGs and AGNs, excluding from further consideration galaxies located between the two lines. The excluded objects are a minor fraction: $\sim 10 \%$ of void galaxies and $\sim 16 \%$ of cluster galaxies. Thus this exclusion cannot significantly bias our results. This classification yielded 588 cluster AGNs, 118 void AGNs, 1077 cluster SFGs and 902 void SFGs.

To check the stability of our classification we have reclassified our sources using the criterion by Lara-López et al. (2010) which exploits a different set of emission lines $(\mathrm{H} \alpha,[\mathrm{NII}]$ and $[\mathrm{SII}])$. We got the same classification for $\simeq 97 \%$ of void and $\simeq 82 \%$ of cluster SFGs, and for $\simeq 83 \%$ void and $\simeq 85 \%$ cluster AGNs.

We note that while the adopted classification proved to be solid and free from contamination by objects of uncertain type, there is a price to pay for that: we are missing galaxies with significant contributions from both nuclear activity and star-formation, which are located in the intermediate region between pure SFGs and pure AGNs in the BPT diagram; however, as already mentioned above, they are a small fraction of the initial sample.

We are also missing strongly obscured AGNs which may fall in the SFG region of the diagram as well as dust-enshrouded SFGs which do not show emission lines. However only a small fraction of AGNs are heavily obscured $(7.6(+1.1,-2.1) \%$; Ricci et al. 2015), and the mean AGN fraction in the local universe is substantially 


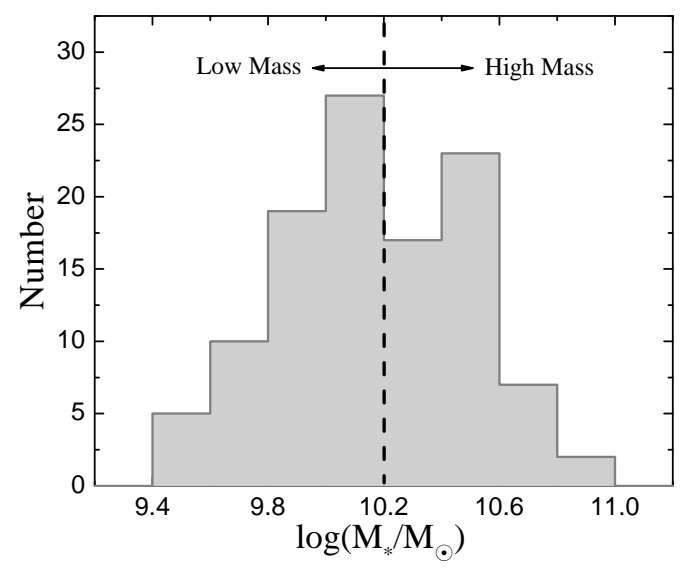

Figure 2. Distribution of stellar masses for our samples defined in Section 2. The vertical dashed line shows the boundary between low stellar mass (LSM) and high stellar mass (HSM) sub-samples. The distribution is the same for our four source groups: SFGs (AGNs) in clusters and in voids (see text).

lower than that of star-forming galaxies, as shown by the many references on that cited in Section 1. The fraction of dust-enshrouded SFGs is also very small (Hwang \& Geller 2013). We can thus safely assume that both source types are too rare to significantly affect our results.

As mentioned in Section 1, it is widely agreed that $F_{\mathrm{AGN}}$ strongly correlates with stellar mass, $M_{\star}$, so that the effect of environment must be investigated at fixed $M_{\star}$. To this end to compare the properties of SFGs and AGNs in clusters and in voids in a way as homogeneous as possible, AGN and SFG galaxies in both environments were randomly drawn from the parent samples in such a way to have the same number of objects in each stellar mass bin so that the stellar mass distribution is the same for both populations (Fig. 2). Each sample has been subdivided in two stellar mass ranges containing similar numbers of galaxies: $9.4 \leq \log \left(M_{\star} / M_{\odot}\right)<10.2$ (low stellar mass; LSM, 60 galaxies per subsample) and $10.2 \leq \log \left(M_{\star} / M_{\odot}\right) \leq 11.0$ (high stellar mass; HSM, 49 objects per subsample). Galaxy properties come from the MPA/JHU catalog which includes accurate aperture corrections (Brinchmann et al. 2004). For AGNs, SFRs could not be estimated from classical SFR diagnostics like the $\mathrm{H} \alpha$ luminosity because it is affected by the AGN component. Therefore the $\mathrm{D}_{n} 4000$ values were used instead. In turn, the star-formation contribution affects the strengths of AGN lines, diluting their ratios. This however does not affect our analysis since we are using line ratios only to select AGNs and the use of the conservative Kewley et al. (2001) limit ensures that our AGN sample is clean.

\section{RESULTS}

The four upper panels of Fig. 3 contrast the distributions of specific SFRs (sSFRs, i.e. SFRs per unit stellar mass) in clusters and in voids for SFGs and AGNs subdivided into LSM and HSM galaxies. The distributions for field SFGs and AGNs in the same ranges of redshift and stellar mass (control samples) are also
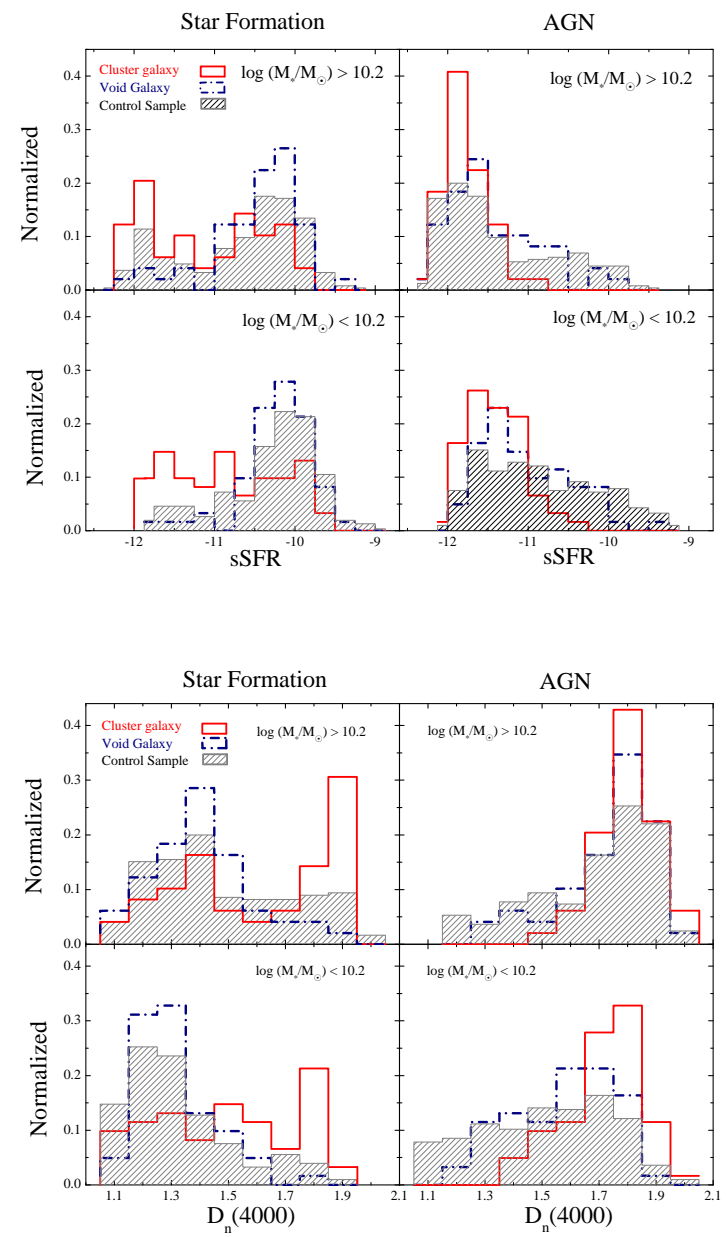

Figure 3. Distribution of specific SFRs (sSFRs; upper panel) and $\mathrm{D}_{n} 4000$ (lower panel) for SFGs and AGNs in clusters (red solid line) and voids (blue dash-dot line), subdivided in two bins of stellar mass compared to the control sample (gray).

shown for comparison. The control samples where built randomly selecting from the SDSS DR7 catalog galaxies with the same criteria used for our SFG and AGN samples, excluding those in clusters and in voids. The selected galaxies were then classified as SFGs or AGNs in the same way as void and cluster galaxies. In this way we obtained SFG and AGN control samples five times larger than void and cluster samples, i.e. containing 545 galaxies each. In both stellar mass ranges the distribution of sSFRs of cluster SFGs galaxies is broad and extends to low values, consistent with the notion that the central regions of clusters are populated by red galaxies in essentially passive evolution, while star-forming late-type galaxies populate the cluster outskirts. The red, passive population is rare in voids, whose sSFR distribution is concentrated at relatively large values. The distribution of the control sample is intermediate between those in clusters and in voids (although somewhat closer to that in voids) for the HSM galaxies but much closer to that in voids for LSMs. A quantitative comparison is provided by the probabilities of the null hypothesis (same parent distribution for different SFG samples) for void/cluster, void/control sample and cluster/control sample SFGs given by the Kolmogorov-Smirnov (KS) test, listed in 
Table 1

KS probabilities, $P$, that distributions of sSFRs or of $\mathrm{D}_{n} 4000$ for different sample pairs of SFGs and AGN galaxies are drawn from the same parent population. $P=0.0$ means that the probability is $<0.001$.

\begin{tabular}{llcccc}
\hline \hline & & \multicolumn{2}{c}{ LSM } & \multicolumn{2}{c}{ HSM } \\
& & sSFR & $\mathrm{D}_{n} 4000$ & $\mathrm{sSFR}$ & $\mathrm{D}_{n} 4000$ \\
\hline \multirow{3}{*}{ SFG } & Void-Cluster & 0.0 & 0.0 & 0.0 & 0.0 \\
& Void-Control & 0.355 & 0.916 & 0.089 & 0.052 \\
& Cluster-Control & 0.0 & 0.0 & 0.016 & 0.008 \\
& & & & & \\
& Void-Cluster & 0.002 & 0.0 & 0.002 & 0.147 \\
AGN & Void-Control & 0.014 & 0.041 & 0.147 & 0.052 \\
& Cluster-Control & 0.0 & 0.0 & 0.0 & 0.0 \\
& & & & & \\
\hline
\end{tabular}

Table 1. The distributions of sSFRs of void and cluster AGNs are more similar, with a single peak at quite low sSFRs. However the distributions for both HSM and LSM galaxies in voids have a substantial tail extending to much higher sSFRs than in clusters. Again the distributions of control samples are more similar to those in voids, especially for HSM galaxies; see Table 1 for a quantitative comparison with the KS test.

The lower panels of Fig. 3 carry a similar message. The distributions of $\mathrm{D}_{n} 4000$, a proxy of stellar population age, of cluster SFGs show a peak at large values, indicative of old stellar populations. The peak is more conspicuous and is shifted to somewhat larger values for HSMs, consistent with the "downsizing" scenario according to which the more massive galaxies formed most of their stars earlier on (e.g, Juneau et al. 2005). The distributions in voids peak at substantially lower values of $\mathrm{D}_{n} 4000$, with a shift to higher values (but well below the cluster peak) for HSM galaxies. Like in the case of sSFRs, the distribution for the control sample is intermediate between those in clusters and in voids (but closer to the latter) for HSMs and very similar to that in voids for LSMs (see also Table 1).

Again the distributions are more similar in the case of AGNs, especially for high stellar mass objects. But void galaxies have a conspicuous tail toward low $\mathrm{D}_{n} 4000$ 's, not present in clusters but also seen in the control sample. Almost all AGNs in clusters and most of the HSM ones in voids and in the field are above the boundary (Vergani et al. $2008, \mathrm{D}_{n} 4000=1.5$; e.g.) between spectroscopic lateand early-type galaxies. An independent confirmation of this indication is provided by galaxy colors. Bernardi et al. (2010) showed that early-type (red) and late-type (blue) galaxies occupy different regions of $g-r$ versus $\mathrm{M}_{r}$ color magnitude plane. The boundary between red and blue sequences is $g-r=0.63-0.03\left(\mathrm{M}_{r}+20\right)$, shown by the solid green line in Fig. 4. This figure shows that almost all AGN galaxies in clusters and in voids have early-type colors, i.e. have old stellar populations.

The main result of this paper is illustrated by Fig. 5 which shows the AGN fraction, $F_{\mathrm{AGN}}$, as a function of stellar mass in clusters and in voids. Note that for this comparison we used all cluster and void members, not only the random selection exploited for previous analyses. At lower stellar masses, $F_{\mathrm{AGN}}$ is only slightly but systematically higher in clusters than in voids. Above $10^{10.2} M_{\odot}$ the mass dependence flattens out in voids and steepens in clusters, where it reaches $\simeq 70 \%$ at the high-

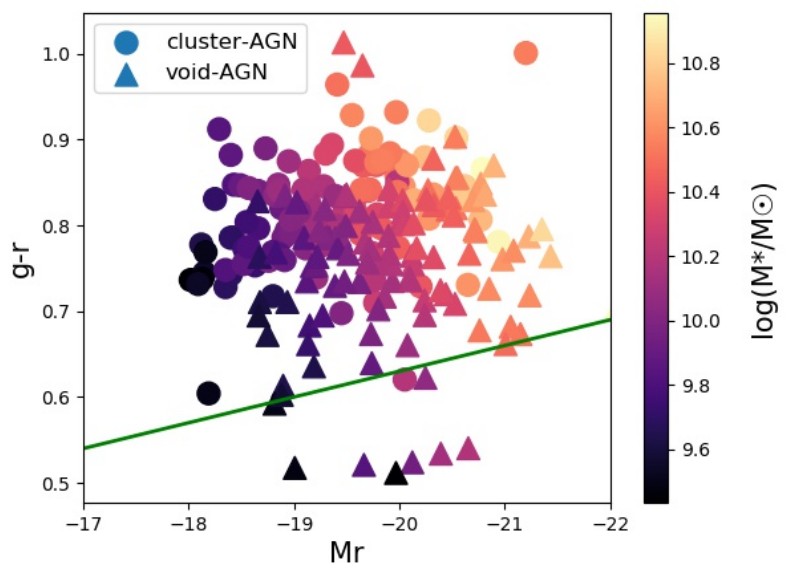

Figure 4. Color-magnitude relation for cluster (circles) and void (triangles) AGN galaxies. The green solid line shows the luminosity dependent threshold, defined by Bernardi et al. (2010), which separates early- and late-type galaxies. Almost all AGN galaxies are redder that the threshold and have therefore early-type colors. Colors of the symbols correspond to the stellar mass according to the scale on the right of the figure.

est stellar masses. The finding of a higher AGN fraction in dense environments is consistent with the results by Manzer \& De Robertis (2014) and by Argudo-Fernández et al. (2018), but disagrees with the results of many other studies (see Sect. 1). The reasons for the disagreements are not totally clear, but it is likely that the different selection criteria have an important role in that. In particular, it is clear from Fig. 5 that the higher AGN fraction in clusters is due, in part, to the higher abundance of massive galaxies that are more likely to host AGNs. The shortage of massive galaxies is specific to voids. The lower panel of Fig. 5 shows that the fraction of SFGs follows the opposite trend to $F_{\mathrm{AGN}}$. In both environments $F_{\mathrm{SFG}}$ increases with decreasing stellar mass down to $\simeq 10^{9.7} M_{\odot}$. At lower stellar masses $F_{\mathrm{SFG}}$ continues to grow in clusters, reaching $100 \%$ at the lowest masses. In the voids, somewhat surprisingly, $F_{\mathrm{SFG}}$ first flattens out and then declines at the lowest masses. Both the increase of the AGN fraction and the decrease of the SFG fraction with increasing stellar mass in dense environments agree with the results by Argudo-Fernández et al. (2018).

Figure 6] shows the average AGN and SFG fractions in clusters versus the distance from the brightest cluster galaxy, assumed to be located close to the cluster center. Again, all cluster members are used for this analysis. The fraction of SFGs increases with increasing distance, consistent with the notion that the sSFR is larger in the outer regions of clusters. The AGN fraction is very weakly dependent on distance, with a slight indication of a decline at the largest distances. This is consistent with the evidences illustrated by Fig. 3 that AGNs are preferentially associated to red galaxies. On the other hand, this result is at odds with Lopes et al. (2017) who claimed a decline of $F_{\text {AGN }}$ with decreasing distance from the cluster center. These authors, however, have only considered massive objects, $\left(\log \left(M_{\star} / M_{\odot}\right)>10.6\right)$.

\section{CONCLUSIONS}




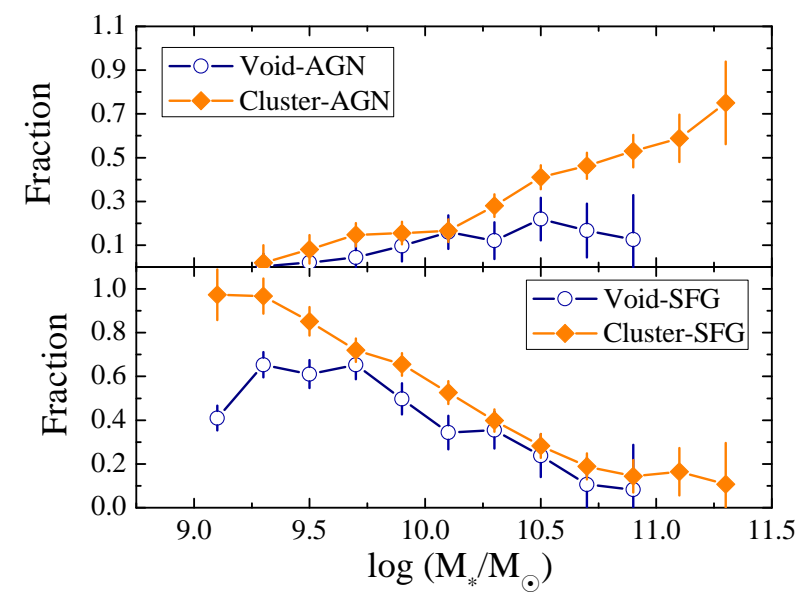

Figure 5. AGN (upper panel) and SFG (lower panel) fractions in clusters and voids as a function of the stellar mass.

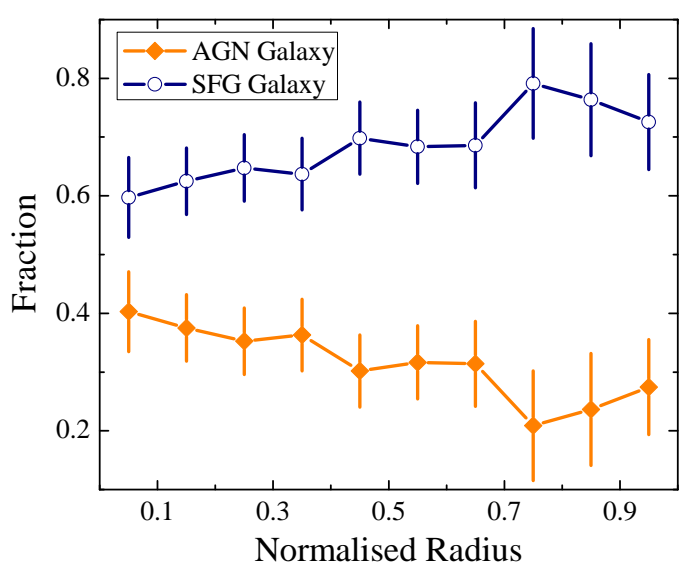

Figure 6. Average AGN and SFG fractions in clusters as a function of the distance from the brightest cluster member.

We have addressed the still unsettled issue of the role of environment on nuclear activity by comparing the fraction of galaxies hosting AGNs in local $(0.01<z<0.04)$ galaxy clusters and voids, as a function of the stellar mass of the host galaxies. To this end, we have exploited volume limited galaxy samples drawn from the void catalogue by Tavasoli et al. (2015) and from the cluster catalogue by Tempel et al. (2014). We have extracted subsamples of star forming galaxies and of galaxies hosting AGNs by means of the classical BPT diagnostics. To be conservative, the assignments to each population were done using the more restrictive among the Kauffmann et al. (2003) and the Kewley et al. (2001) criteria; intermediate galaxies were discarded. The distributions of both sSFRs and $D_{n} 4000$ in clusters and in voids for SFGs differ since clusters host an essentially passive (very low sSFRs), old $\left(\mathrm{D}_{n} 4000 \gtrsim 1.5\right.$, corresponding to spectroscopic early-type galaxies) galaxy population, missing in voids. Such distributions are more similar to each other in the case of galaxies hosting AGNs: both in clusters and in voids they peak at low sSFRs. The distributions of both quantities converge in indicating that both in clusters and in voids galaxies hosting AGNs are generally old, with low star formation activity. This is in keeping with the well established fact that super-massive black holes correlate with spheroidal components (bulges) of galaxies, not with disks (e.g., Kormendy \& Ho 2013). The AGN fraction increases with stellar mass both in clusters and in voids. The mass dependence is, however, quite different: it is mild in voids where $F_{\mathrm{AGN}}$ reaches $\simeq 30 \%$ at most; in clusters it is steeper, particularly above $M_{\star} \simeq 10^{10.1} M_{\odot} ; F_{\mathrm{AGN}}$ reaches $\simeq 70 \%$ at the highest masses. The higher $F_{\mathrm{AGN}}$ in clusters, compared to voids, agrees with the results by Manzer \& De Robertis (2014) and by Argudo-Fernández et al. (2018), but disagrees with the results of many other studies which however have used different selection criteria. The average SFG fraction in clusters is higher in the less dense outer regions, where the fraction of star-forming galaxies is lower. This is consistent with the well known relation between density and morphological type in the local universe (e.g., Dressler 1980): star-forming galaxies prefer low-density environments. The AGN fraction is found to be very weakly dependent on the distance from the cluster center, with a hint of a decrease at the cluster boundary. This finding is at odds with Lopes et al. (2017) who claimed a decline of $F_{\mathrm{AGN}}$ with decreasing distance from the cluster center. Our results indicate that, both in local clusters and in local voids, the nuclear activity is related to galaxy properties such as stellar mass and morphology and is only weakly, if at all, affected by environment. We caution, however, that this conclusion may not apply to intermediate-density systems. For example, it is possible that the extreme environments we have investigated have in common a low merger/interaction rate. In voids close encounters are rare due to the low galaxy density; in clusters merging is ineffective when galaxy velocities are much larger than their stellar velocity dispersions (e.g., Carnevali et al. 1981). The exception are BCGs that cannibalized many satellite galaxies during their lifetime; they are also excluded from our samples.

We thank the anonymous referee for a very thorough review that helped us to significantly improve the quality of this paper and R. van de Weygaert for a fruitful discussion on galaxy evolution in voids. This paper has made use of SDSS data. Funding for the SDSS and SDSS-II has been provided by the Alfred P. Sloan Foundation, the Participating Institutions, the National Science Foundation, the U.S. Department of Energy, the National Aeronautics and Space Administration, the Japanese Monbukagakusho, the Max Planck Society, and the Higher Education Funding Council for England. The SDSS Web site is http://www.sdss.org/. The SDSS is managed by the Astrophysical Research Consortium for the Participating Institutions. The Participating Institutions are the American Museum of Natural History, Astrophysical Institute Potsdam, University of Basel, University of Cambridge, Case Western Reserve University, University of Chicago, Drexel University,Fermilab, the Institute for Advanced Study, the Japan Participation Group, Johns Hopkins University, the Joint Institute for Nuclear Astrophysics, the Kavli Institute for Particle Astrophysics and Cosmology, the Korean Scientist Group, the Chinese Academy of Sciences (LAMOST), Los Alamos Na- 
tional Laboratory, the Max-Planck-Institute for Astronomy (MPIA), the MaxPlanck-Institute for Astrophysics (MPA), New Mexico State University, Ohio State University, University of Pittsburgh, University of Portsmouth, Princeton University, the United States Naval Observatory, and the University of Washington.

\section{REFERENCES}

Argudo-Fernández, M., Lacerna, I., \& Duarte Puertas, S. 2018, A\&A, 620, A113

Baldwin, J. A., Phillips, M. M., \& Terlevich, R. 1981, PASP, 93, 5

Baldry, I. K., Balogh, M. L., Bower, R. G., Glazebrook, K., Nichol, R. C., Bamford, S. P. \& Budavari, T. 2006, MNRAS, 373,469

Brinchmann, J., Charlot, S., White, S. D. M., et al. 2004, MNRAS, 351, 1151

Bernardi, M., Shankar, F., Hyde, J. B., Mei, S., Marulli, F., and Sheth, R. K.2010, MNRAS, 404, 2087

Carnevali, P., Cavaliere, A., \& Santangelo, P. 1981, ApJ, 249, 449

Carter, B. J., Fabricant, D. G., Geller, M. J., Kurtz, M. J., \& McLean, B. 2001, ApJ, 559, 606

Constantin, A., Hoyle, F., \& Vogeley, M. S. 2008, ApJ, 673, 715

Deng, X.-F., Song, J., Chen, Y.-Q., Jiang, P., \& Ding, Y.-P. 2012, ApJ, 753, 166

Dressler, A. 1980, ApJ, 236, 351

Ellison, S. L., Patton, D. R., Mendel, J. T., \& Scudder, J. M. 2011, MNRAS, 418, 2043

Ellison, S. L., Patton, D. R., \& Hickox, R. C. 2015, MNRAS, 451, L35

Hashimoto, Y., Oemler, A., Jr., Lin, H., \& Tucker, D. L. 1998, ApJ, 499, 589

Hwang, H. S., \& Geller, M. J. 2013, ApJ, 769, 116
Karhunen, K., Kotilainen, J. K., Falomo, R., \& Bettoni, D. 2014, MNRAS, 441, 1802

Juneau S. et al., 2005, ApJ, 619, L135

Kauffmann, G., et al. 2003, MNRAS, 341, 33

Kauffmann, G., et al. 2004, MNRAS, 353, 713

Kewley, L. J., Dopita M. A.,Sutherland R. S., Heisler C. A, \& Trevena J. 2001, ApJ, 556, 121

Kormendy, J., \& Ho, L. C. 2013, ARA\&A, 51, 511

Lara-López, M. A., Bongiovanni, A., Cepa, J., et al. 2010, A\&A, 519, A31

Lauer TR, Postman M, Strauss MA, Graves GJ \& Chisari NE. 2014, ApJ, 797, 82

Lopes, P. A. A., Ribeiro, A. L. B., \& Rembold, S. B. 2017, MNRAS, 472, 409

Manzer, L. H., \& De Robertis, M. M. 2014, ApJ, 788, 140

Miller, C. J., Nichol, R. C., Gómez, P. L., Hopkins, A. M., \& Bernardi, M. 2003, ApJ, 597,142

Oemler, A., Jr. 1974, ApJ, 194, 1

Pimbblet, K. A., Shabala, S. S., Haines, C. P., Fraser-McKelvie, A., \& Floyd, D. J. E. 2013, MNRAS, 429, 1827

Ricci, C., Ueda, Y., Koss, M. J., et al. 2015, ApJ, 815, L13

Sabater, J., Best, P. N., \& Heckman, T. M. 2015, MNRAS, 447, 110

Satyapal, S., Ellison, S. L., McAlpine, W., et al. 2014, MNRAS, 441,1297

Scott C., Kaviraj S., 2014, MNRAS, 437, 2137

Schawinski, K., Dowlin, N., Thomas, D., Urry, C. M., \& Edmondson, E. 2010, ApJ, 714, L108

Tavasoli, S., Rahmani, H., Khosroshahi, H. G., Vasei, K., \&

Lehnert, M. D. 2015, ApJ, 803, L13

Tempel,E. et al. 2014,A\&A, 566,A1

Vergani, D., et al. 2008, A\&A, 487, 89

Yang, G., et al. 2018, MNRAS, 480, 1022 